\title{
On the Use of Evolutionary Programming for Combinational Logic Circuits Design
}

\author{
Marco A. Contreras-Cruz, Victor Ayala-Ramirez, Paola B. Alvarado-Velazco \\ Electronics Engineering Department, Universidad de Guanajuato, DICIS \\ Carr. Salamanca-Valle de Santiago, Km. 3.5+1.8, 36700, Salamanca, Mexico \\ \{macc,palvarado\}@laviria.org, ayalav@ugto.mx
}

\begin{abstract}
In this paper, we propose a new methodology to solve the combinational logic circuit design problem by using evolutionary programming. We have implemented a set of evolutionary operators to evolve combinational circuits. Our method uses a set of examples described by their truth table, and it automatically generates a combinational logic function that represents this set. We test our system by using some examples from the literature, and also, we present a set of examples addressing more complex problems to validate the performance of our method.
\end{abstract}

Keywords: combinational logic, evolutionary programming, automated circuit design.

\section{Introduction}

Combinational logic design is an interesting problem for digital electronics. Given the availability of fully programmable devices like Field Programmable Gate Arrays (FPGAs), the automatic synthesis of digital circuits is an important task, specifically when they use several input variables that make difficult the optimization of the circuit by a human designer.

There are mainly two classical approaches to solve the design of digital circuits satisfying a given specification: i) using graphical methods like Karnaugh Maps and, ii) using automated algorithms like the Quine-McCluskey method 1]. In both cases, they are well suited for synthesis problems where there are a small number of input variables. Several authors have also proposed to use Evolutionary Computation (EC) techniques to solve the combinational logic design problem. Among them, we can find works using Particle Swarm Optimization (PSO) 2], Genetic Programming (GP) 3456], and Evolution Strategies (ES) [7] among some others. These authors have used diverse optimization criteria for the design of digital circuits.

We propose to solve the problem with another evolutionary technique, Evolutionary Programming (EP). The main advantage of EP is that we can represent individuals with variable length in a simple way. The proposed work describes the details of how to configure the EP elements to solve the combinational logic design problem. To validate the performance of our system, we tested it with a

E. Bayro-Corrochano and E. Hancock (Eds.): CIARP 2014, LNCS 8827, pp. 191198 2014.

(C) Springer International Publishing Switzerland 2014 
set of examples from the literature, and we also present a set of more complex problems with respect to the number of input variables.

The rest of this paper is organized as follows: Section 2 includes the formulation of the combinational logic circuit design in terms of our approach. The tests are presented in Section 3, in Section 4 we show our results, and finally we present in Section 5 our main conclusions and findings.

\section{Digital Realization of Combinational Logic}

In our approach, we take the truth table of a digital system as input. The goal of the developed system is to find a digital circuit that realizes such a specification. The simpler way to implement combinational logic is by using two gates level, either by using a Sum of Products (SOP) realization or using a Product of Sums (POS) implementation. Both representations are known as the canonical forms, and by using DeMorgan's theorem, six other canonical forms can be generated 8. For our work, we have used both SOP and POS canonical forms.

\subsection{Evolutionary Programming}

The EP is an extension of the Genetic Algorithms where individuals can be encoded using variable length representations. A distinctive feature of the EP is that only uses mutation operators to evolve the population. The general structure of the EP is shown in Algorithm [1].

\subsection{Representation and Initialization of Individuals}

We use a variable representation to code a combinational logic function similar to the one presented in [10. In our work, an individual of the population is a feasible logical function that represents a set of $n_{e}$ examples in a design problem of $n$ input variables. A logical function $L$ is a set of $\mathrm{AND}_{i}$ gates, $i=1, \ldots, G$ of the input variables $x_{j}, j=1, \ldots, n$. Each input variable can be used in three mutually exclusive forms: the variable is used either in its positive form (represented as $x_{j}$ ), or in its negative form (represented as $x_{j}^{\prime}$ ). The third alternative is not to use the input variable in that gate (represented as \{\} ).

To initialize each individual in the population, we generate a random number of AND gates between 1 and $G_{\text {max }}$. Subsequently, we assign to each AND gate a random logic selecting between positive or negative forms for each input variable.

\subsection{Individual Evaluation}

We formulate the design of logical functions like a classification task, where a logical function is a classifier. To evaluate the performance of the logical function, we consider classification errors expressed as false positives $(F P)$ and false negatives $(F N)$. 


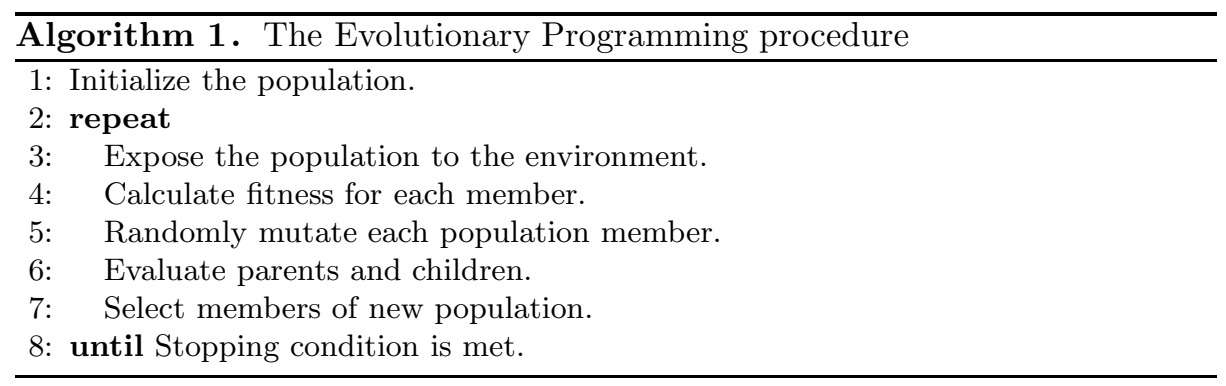

In Equation 1, we present the error function. For our representation of the logical function, we can handle cases of $F N$, so, we penalize the $F P$ cases with a penalization factor $n_{e}$.

$$
E=F N+n_{e} F P
$$

The optimal combinational circuit exhibits no classification errors for all the input combinations in the truth table and simultaneously, it uses the minimal number of gates in its design. The latter issue is addressed by Equation 2, where the cost $C$ of a logical function is the sum of the costs per gate, and the cost $C_{i}$ of each gate is the number of its active inputs $n_{i}$ (input variables in their positive or negative form).

$$
C=\sum_{i} C_{i}
$$

In order to simultaneously satisfy both constraints, we put them together in a single evaluation function (Equation 3). This function favors logic functions that produce less classification errors, by using a factor $f_{p}$ (fixed in two). We have normalized the cost by using a maximum cost $C_{\max }=(n+1) \times G_{\max }$.

$$
F(L)=f_{p} E+\frac{C}{C_{\max }}
$$

The goal of the evolution process is then to find $L^{*}$ that minimizes the Equation 3.

As our selection method, we have used elitist selection mechanism. We retain the fifty percent of the best individuals of the population (between parents and children) for the next generation. The remaining fifty percent is filled with some mutated individuals.

\subsection{Mutation Operators}

In EP, mutation is the enabling mechanism of the evolution process. Each logical function in the population is used to generate a new one by applying some mutation operators. In this work, seven mutation operations have been implemented: to add, to delete, and to update an input variable; to add, to delete, and 
to disaggregate a gate and to unite two gates. The description of each mutation operator is presented below.

- Delete input variable. Many times, an input variable is not necessary to get the desired output value in a combinational logic function. This operator selects at random an input variable from an AND gate and removes it.

- Add input variable. This operator selects at random an AND gate in the logical function, finds the set of missing input variables, selects at random one of them to be added either in its positive or negative form.

- Update input variable. Update operator modifies the logic of an input variable, randomly selected from an AND gate, by changing it to its complemented form.

- Add gate. This operator adds an AND gate to the logical function by using an example of the truth table (a minterm).

- Delete gate. The working of this operator is to select at random an AND gate of the logical function and then to remove it.

- Disaggregate gate. The disaggregate operator separates an AND gate into two AND gates that cover the same combinations of the truth table except for a single minterm. In Figure 1a, we describe this operator. It selects at random an AND gate of a logical function, finds the missing set of input variables, creates two AND gates by adding at random a missing variable, and finally removes the selected gate.

- Unite two gates. This operator joins two AND gates into another gate that uses only the common input variables. This operator tries to cover more asserted combinations in the truth table, similar to the implicant concept. In Figure $1 \mathrm{~b}$ the application process of this operator is presented.

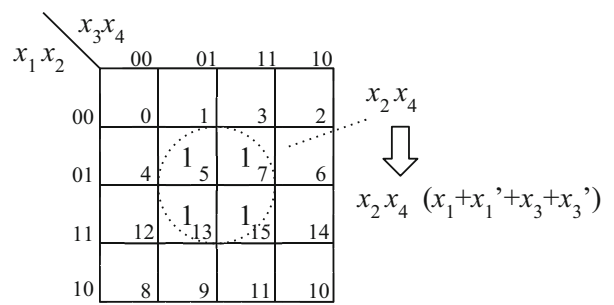

(a) Disaggregate operator.

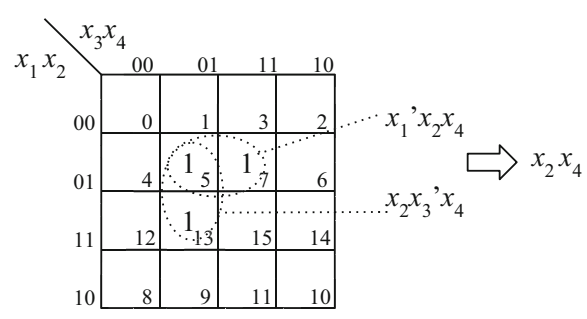

(b) Unite operator.

Fig. 1. Disaggregate and unite operators. The disaggregate operator generates two gates by adding a different missing input variable whilst the unite operator tries to join two gates that have common input variables.

\section{Tests Protocol}

We have performed two tests to validate our system. On one hand, we test the performance of the evolutionary synthesizer in a set of problems presented in the literature; this problem uses a small number of input variables. On the other hand, in the second experiment, we address the solution of a set of problems using 10 input variables. This implies a larger complexity to find the solution. 
Table 1. First set of examples to be synthesized. List of minterms.

\begin{tabular}{ccc}
\hline Inputs Outputs & Function \\
\hline 4 & 1 & $f_{1}=\sum(1,2,4,5,7,8,10,11,13,14)$ \\
4 & 1 & $f_{2}=\sum(2,3,8,9,11,12,13,14)$ \\
5 & 1 & $f_{3}=\sum(3,5,6,9,10,12,17,18,20,24)$ \\
4 & 2 & $f_{4,1}=\sum(0,1,2,4,5,8)$ \\
& & $f_{4,2}=\sum(11,14,15)$ \\
5 & 3 & $f_{5,1}=\sum(0,1,2,3,4,5,6,7,8,9,10,11,12,13,14,15,28,29,30,31)$ \\
& & $f_{5,2}=\sum(0,2,4,6,7,8,10,12,14,15,16,18,20,22,23,24,26,28,30,31)$ \\
& & $f_{5,3}=\sum(4,5,12,13,20,21,28,29)$ \\
\hline
\end{tabular}

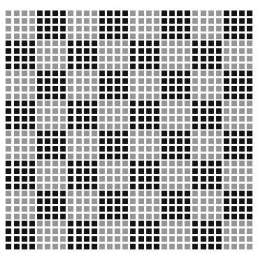

(a) Chessboard.

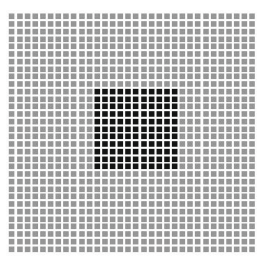

(b) Square.

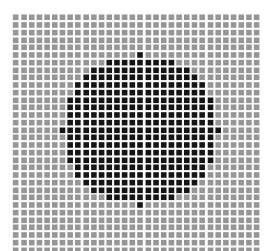

(c) Circle.

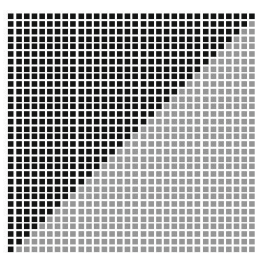

(d) Comparator.

Fig. 2. Second set of examples. The truth tables are represented on the Cartesian plane. The black dots are the "true" ("1") values in the truth table whilst the gray dots represent the "false" ("0") values in the truth table.

Experiment 1. We have used a set of five examples presented by Coello et al. 2. These examples are design problems with four and five input variables. In Table 1, we show the problems that were solved.

Experiment 2. We have tested our method on four design problems of a larger complexity (10 input variables). We display their truth tables using a Cartesian plane (see Figure 2) where the 5 more significant bits are represented in the $y$-axis and the 5 least significant bits are represented in the $x$-axis.

\section{Results}

The evolutionary system for the automatic design of combinational logic circuits was implemented in the $\mathrm{C}$ language, on an Intel Core i5 processor running at 2.5 $\mathrm{GHz}$ with $8 \mathrm{~GB}$ of RAM memory. Given its stochastic formulation, the system was tested 30 times for each design problem. In both experiments the parameters are: a maximum of 50 gates $\left(G_{\max }\right), 10$ individuals, and 5000 generations. Convergence is tested every 100 generations.

Experiment 1. Our system has generated the same logical function as a human expert for all the problems in this set. Table 2 shows the statistical results for the 
Table 2. Results of the Experiment 1 using SOP and POS representation. Statistical results of the generated logical functions by our evolutionary synthesizer for each test are showed. The data * represent the optimal cost computed by the Karnaugh Map method.

\begin{tabular}{|c|c|c|c|c|c|c|}
\hline \multirow{2}{*}{ Function } & \multirow{2}{*}{ Method } & \multicolumn{2}{|c|}{ Realization cost } & \multicolumn{3}{|c|}{ Search cost (s) } \\
\hline & & mean & std & best & mean & std \\
\hline \multirow{2}{*}{$f_{1}$} & SOP & $32 / 32^{*}$ & 0 & 0.1540 & 0.1856 & 0.0217 \\
\hline & POS & $30 / 30^{*}$ & 0 & 0.0053 & 0.0802 & 0.0053 \\
\hline \multirow{2}{*}{$f_{2}$} & SOP & $15 / 15^{*}$ & 0 & 0.0570 & 0.0732 & 0.0141 \\
\hline & POS & $15 / 15^{*}$ & 0 & 0.0055 & 0.0685 & 0.0055 \\
\hline \multirow{2}{*}{$f_{3}$} & SOP & $60 / 60^{*}$ & 0 & 0.2570 & 0.3038 & 0.0389 \\
\hline & POS & $66 / 65^{*}$ & 1 & 0.1843 & 1.1229 & 0.1843 \\
\hline \multirow{2}{*}{$f_{4,1}$} & $\overline{\mathrm{SOP}}$ & $11 / 11^{*}$ & $\overline{0}$ & 0.0480 & 0.0530 & 0.0034 \\
\hline & POS & $15 / 15^{*}$ & 0 & 0.0062 & 0.0762 & 0.0062 \\
\hline \multirow{2}{*}{$f_{4,2}$} & SOP & $8 / 8^{*}$ & $\overline{0}$ & 0.0330 & 0.0409 & 0.0031 \\
\hline & POS & $5 / 5^{*}$ & 0 & 0.0174 & 0.0765 & 0.0174 \\
\hline \multirow{2}{*}{$f_{5,1}$} & $\mathrm{SOP}$ & $4 / 4^{*}$ & 0 & 0.0960 & 0.1505 & 0.0351 \\
\hline & POS & $6 / 6^{*}$ & 0 & 0.0417 & 0.1122 & 0.0417 \\
\hline \multirow{2}{*}{$f_{5,2}$} & SOP & $4 / 4^{*}$ & 0 & 0.0760 & 0.1280 & 0.0522 \\
\hline & POS & $6 / 6^{*}$ & 0 & 0.0286 & 0.1169 & 0.0286 \\
\hline \multirow{2}{*}{$f_{5,3}$} & $\overline{\mathrm{SOP}}$ & $2 / 2^{*}$ & 0 & 0.0650 & 0.0756 & 0.008 \\
\hline & POS & $2 / 2^{*}$ & 0 & 0.0429 & 0.0992 & 0.0429 \\
\hline
\end{tabular}

Table 3. Results of the Experiment 2. Statistical results of the generated logical functions by our evolutionary synthesizer for each test using both representations: SOP and POS.

\begin{tabular}{|c|c|c|c|c|c|c|}
\hline \multirow{2}{*}{ Function } & \multirow{2}{*}{ Method } & \multicolumn{2}{|c|}{ Realization cost } & \multicolumn{3}{|c|}{ Search cost (s) } \\
\cline { 3 - 7 } & & mean & std & best & mean & std \\
\hline \multirow{2}{*}{$f_{\text {chess }}$} & SOP & $\mathbf{6 . 0 0 0 0}$ & 0.0000 & $\mathbf{2 . 4 1 0 0}$ & 4.8967 & 5.2330 \\
& POS & $\mathbf{6 . 0 0 0 0}$ & 0.0000 & 2.5070 & $\mathbf{3 . 6 7 4 5}$ & 1.1860 \\
\hline \multirow{2}{*}{$f_{\text {square }}$} & SOP & 123.2333 & 1.9597 & 130.3380 & 212.0314 & 31.3882 \\
& POS & $\mathbf{3 6 . 1 3 3 3}$ & 0.3457 & $\mathbf{5 1 . 0 3 1 0}$ & $\mathbf{7 9 . 4 2 1 7}$ & 18.0267 \\
\hline \multirow{2}{*}{$f_{\text {circle }}$} & SOP & 273.3000 & 12.3516 & $\mathbf{7 4 5 . 9 2 3 0}$ & $\mathbf{1 0 5 8 . 3 9 4 3}$ & 135.2601 \\
& POS & $\mathbf{2 3 0 . 0 3 3 3}$ & 8.9384 & 884.3140 & 1408.0725 & 228.7747 \\
\hline \multirow{2}{*}{$f_{\text {cmp }}$} & SOP & 286.7000 & 7.7999 & 1350.4690 & 2069.2972 & 286.8293 \\
& POS & $\mathbf{2 0 0 . 8 3 3 3}$ & 5.8844 & $\mathbf{4 9 6 . 6 2 7 0}$ & $\mathbf{7 4 5 . 5 0 5 4}$ & 96.2671 \\
\hline
\end{tabular}

Table 4. The best cost generated by the two methods of representation for each test

\begin{tabular}{ccccc}
\hline Method & $\mathbf{f}_{\text {chess }}$ & $\mathbf{f}_{\text {square }}$ & $\mathbf{f}_{\text {circle }}$ & $\mathbf{f}_{\mathbf{c m p}}$ \\
\hline SOP & $\mathbf{6}$ & 120 & 255 & 276 \\
POS & $\mathbf{6}$ & $\mathbf{3 6}$ & $\mathbf{2 1 5}$ & $\mathbf{1 9 4}$ \\
\hline
\end{tabular}

realization cost and for the computational search cost to generate the optimal solution using SOP and POS realizations. 
Experiment 2. Table 3 summarizes the statistical results of the second experiment for both types of realizations (SOP and POS). In all test cases the optimal POS realization is synthesized spending lower search cost. The optimal SOP representation has been only attained in the $f_{\text {chess }}$ test case (see Table 4). The proposed method generates logical functions with a realization cost very similar to the cost of the optimal logical functions. For example, optimal SOP realization costs for the $f_{\text {square }}$ and $f_{c m p}$ test cases are 120 and 271 , respectively. Our approach synthesizes realizations with average costs of 123.2 and 286.7, respectively. Time spent data shows that the $f_{\text {circle }}$ is the most difficult problem in the test set. However, the proposed approach generates a correct logical circuit in all the test cases.

\section{Conclusion}

We proposed a new methodology to generate an automated synthesizer of combinational logic circuits; we have posed the combinational logic circuits design as a classification task. Our approach uses a canonical representation of the resulting circuit. We also proposed a set of mutation operators that enables the automated design of the logical functions. Our method was tested using several problems with incremental complexity. An advantage of the proposed method is that the logical functions generated by our evolutionary design system can be easily implemented in Field Programmable Gate Array systems. The optimal representation (Sum of Products or Product of Sums) depends on the specific problem being solved. As a perspective of future work, we intend to develop better evaluation schemes to reduce the time needed in the search process, in order to avoid the exhaustive test of truth table rows. Another direction of our future work is the exploration of multi-level gate implementations.

Acknowledgments. Marco A. Contreras-Cruz would like to acknowledge CONACYT for the financial support through the educational scholarship number 568675/302121.

\section{References}

1. Morris Mano, M., Kime, C.: Logic and Computer Design Fundamentals, 4th edn. Prentice Hall (2007)

2. Coello Coello, C.A., Luna, E.H., Aguirre, A.H.: A comparative study of encodings to design combinational logic circuits using particle swarm optimization. In: Proc. 2004 NASA/DoD Conference on Evolvable Hardware, pp. 71-78. IEEE (2004)

3. Miller, J.F., Job, D., Vassilev, V.K.: Principles in the evolutionary design of digital circuits-Part I. Genetic Programming and Evolvable Machines 1(1-2), 7-35 (2000)

4. Vasicek, Z., Sekanina, L.: On area minimization of complex combinational circuits using Cartesian genetic programming. In: Proc. 2012 IEEE Congress on Evolutionary Computation (CEC), pp. 1-8. IEEE (2012) 
5. Rajaei, A., Houshmand, M., Rouhani, M.: Optimization of combinational logic circuits using NAND gates and genetic programming. In: Gaspar-Cunha, A., Takahashi, R., Schaefer, G., Costa, L. (eds.) Soft Computing in Industrial Applications. AISC, vol. 96, pp. 405-414. Springer, Heidelberg (2011)

6. Karakatic, S., Podgorelec, V., Hericko, M.: Optimization of combinational logic circuits with genetic programming. Electronics and Electrical Engineering 19(7), 86-89 (2013)

7. Stomeo, E., Kalganova, T., Lambert, C., Lipnitsakya, N., Yatskevich, Y.: On evolution of relatively large combinational logic circuits. In: Proc. 2005 NASA/DoD Conference on Evolvable Hardware, pp. 59-66. IEEE (2005)

8. Sandige, R.: Top-down design process for gate-level combinational logic design. IEEE Transactions on Education 35(3), 247-252 (1992)

9. Kennedy, J., Kennedy, J.F., Eberhart, R.C.: Swarm intelligence. Morgan Kaufmann (2001)

10. Zebulum, R.S., Vellasco, M.S., Pacheco, M.A.: Variable length representation in evolutionary electronics. Evolutionary Computation 8(1), 93-120 (2000) 